\title{
Cigarette smoke impairs airway epithelial barrier function and cell-cell
}

\section{contact recovery}

\author{
I.H. Heijink*\#, S.M. Brandenburg*, D.S. Postma", and A.J.M. van Oosterhout*,?
}

ABSTRACT: Cigarette smoking, the major cause of chronic obstructive pulmonary disease (COPD), induces aberrant airway epithelial structure and function. The underlying mechanisms are unresolved so far.

We studied effects of cigarette smoke extract (CSE) on epithelial barrier function and wound regeneration in human bronchial epithelial 16HBE cells and primary bronchial epithelial cells (PBECs) from COPD patients, nonsmokers and healthy smokers.

We demonstrate that CSE rapidly and transiently impairs 16HBE barrier function, largely due to disruption of cell-cell contacts. CSE induced a similar, but stronger and more sustained, defect in PBECs. Application of the specific epidermal growth factor receptor (EGFR) inhibitor AG1478 showed that EGFR activation contributes to the CSE-induced defects in both 16HBE cells and PBECs. Furthermore, our data indicate that the endogenous protease calpain mediates these defects through tight junction protein degradation. CSE also delayed the reconstitution of 16HBE intercellular contacts during wound healing and attenuated PBEC barrier function upon wound regeneration. These findings were comparable between PBECs from smokers, healthy smokers and COPD patients.

In conclusion, we demonstrate for the first time that CSE reduces epithelial integrity, probably by EGFR and calpain-dependent disruption of intercellular contacts. This may increase susceptibility to environmental insults, e.g. inhaled pathogens. Thus, EGFR may be a promising target for therapeutic strategies to improve mucosal barrier function in cigarette smoking-related disease.

KEYWORDS: Cell adhesion molecules, cigarette smoking, epithelial cell biology, epithelial repair, lung epithelial permeability, pathogenesis of chronic obstructive pulmonary disease

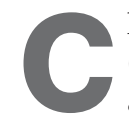

hronic obstructive pulmonary disease (COPD) is an inflammatory airway disease associated with airway remodelling and/ or lung emphysema, leading to accelerated lung function decline. Although smoking is the major risk factor for COPD, it has remained unclear how different COPD phenotypes develop from the same cigarette smoke exposure. Inhaled tobacco smoke first encounters the airway epithelium, which forms a highly regulated barrier. Under pathological conditions, disruption of this barrier may lead to increased access of pollutants and pathogens to the submucosa. Epithelial barrier function is maintained by tight junctions (TJs), which comprise the interacting proteins occludin, zona occludens (ZO)-1 and claudins. TJs restrict paracellular permeability and fulfil a fence function, keeping the basolateral region separated from the apical region. Efficient TJ restoration upon wounding may be crucial for epithelial homeostasis.

Smoking may considerably disturb epithelial junctions by inducing structural changes, such as mucous hyperplasia in the airways of COPD patients [1]. Smoking is not only associated with increased numbers of mucus-producing cells [2, 3], but also with increased mucosal permeability [4] and increased permeability to allergens in vitro [5, 6]. Similarly, cigarette smoke may facilitate transepithelial crossing of viruses and bacteria, with crucial consequences, since bacterial infections have been implicated in nearly $50 \%$ of COPD exacerbations [7]. Therefore, it is important that the mechanisms involved in smoke-induced barrier dysfunction are understood.

\section{AFFILIATIONS}

*Laboratory for Allergology and Pulmonary Diseases, Dept of Pathology and Medical Biology, \#Dept of Pulmonology, GRIAC Research Institute, University Medical Center Groningen, University of Groningen, Groningen, The Netherlands.

"Senior authorship is shared by these authors.

\section{CORRESPONDENCE}

I.H. Heijink

University Medical Center Groningen University of Groningen

Hanzeplein 1

NL-9713 GZ

Groningen

The Netherlands

E-mail: h.i.heijink@int.umcg.nl

Received:

Dec 152010

Accepted after revision:

June 272011

First published online:

July 202011 
The smoke-induced increase in permeability was initially attributed to the cytotoxic effects of smoke. More recently, cigarette smoking, as well as 6-day exposure to cigarette smoke extract (CSE) in vitro, has been shown to cause downregulation of apical junctional complex genes in airway epithelium [8]. Furthermore, mainstream cigarette smoke reduces transepithelial resistance and induces tyrosine phosphorylation of occludin in human airway adenocarcinoma Calu-3 cells $[9,10]$. Additionally, cigarette smoke condensate causes damage to human bronchial epithelial BEAS-2B cells by a mechanism involving activation of the epidermal growth factor receptor (EGFR)/extracellular signal-related kinase (ERK) pathway and subsequent delocalisation of TJ proteins [11]. This effect could be attributed to lipophilic compounds present in CSE (including phenolic compounds, aldehydes and polycyclic aromatic hydrocarbons) that are able to pass the epithelial plasma membrane [12]. Effects of CSE on epithelial resistance as a read-out for barrier function have not been investigated. Our goal was to evaluate the effect of a non-toxic concentration of CSE on TJ formation and epithelial barrier function and to elucidate the underlying mechanism of smoke-induced epithelial barrier dysfunction.

We studied effects of CSE exposure in human bronchial epithelial cell line $16 \mathrm{HBE}$ as well as in primary bronchial epithelial cells (PBECs) from COPD patients and healthy nonsmoking and smoking donors. We demonstrate that CSE induces a transient defect in epithelial barrier function and decreases reconstitution of $16 \mathrm{HBE}$ cell-cell contacts upon wounding, probably by EGFRdependent disruption of TJs. To our knowledge, this study shows for the first time that CSE induces comparable defects in PBEC, but with a delayed onset and a prolonged period of recovery. No clear differences in the response to wounding and CSE were observed between PBECs from nonsmokers, healthy smokers and COPD patients.

\section{METHODS}

\section{Epithelial cell culture}

$16 \mathrm{HBE}$ cells were kindly provided by D.C. Gruenert (University of California, San Francisco, CA, USA) and cultured as described previously [13]. PBECs were obtained from three severe COPD patients with Global Initiative for Chronic Obstructive Lung Disease (GOLD) stages III and IV disease [14] by bronchial brushings (inclusion based on $\geqslant 10$ pack-yrs smoking, forced expiratory volume in $1 \mathrm{~s}$ (FEV1)/forced vital capacity $<70 \%$ and FEV $1<50 \%$ of predicted and age $50-62$ yrs; table 1 ) according to standard guidelines [15]. The medical ethics committee of the University Medical Center Groningen, Groningen, the Netherlands, approved the study and participants gave signed informed consent. Additionally, PBECs from four healthy smokers (age 40-48 yrs) and three healthy nonsmokers (age 52-56 yrs) were derived from Lonza (Walkersville, MD, USA). PBECs were grown in $2.5 \mathrm{~mL}$ hormonally supplemented bronchial epithelium growth medium (BEGM; Lonza) on collagen/ fibronectin-coated flasks as described [16], and used for experimentation at passage 2. Before experimentation, cell viability was evaluated by Trypan blue staining (mean viability $95 \pm 0.6 \%$ for brushed cells and $93 \pm 1.6 \%$ for Lonza cells).

Cells were seeded in duplicates into electric cell substrate impedance sensing (ECIS) arrays, chamber slides (LabTek; Thermo Fisher Scientific Inc., Waltham, MA, USA) or 24-well

\begin{tabular}{|c|c|c|c|c|}
\hline TABLE 1 & \multicolumn{4}{|c|}{$\begin{array}{l}\text { Characteristics of the chronic obstructive } \\
\text { pulmonary disease patients from whom epithelial } \\
\text { cells were obtained }\end{array}$} \\
\hline Subject & Age yrs & Sex & $\begin{array}{c}\text { Smoking history } \\
\text { pack-years }\end{array}$ & FEV $1 \%$ pred \\
\hline 1 & 62 & Female & 42 & 18 \\
\hline 2 & 50 & Male & 11 & 20 \\
\hline 3 & 61 & Male & 31 & 48 \\
\hline
\end{tabular}

plates. At $80-90 \%$ confluency, Eagle's minimal essential medium (EMEM) $/ 10 \%$ fetal calf serum (FCS) or BEGM (Lonza) was replaced with EMEM $/ 0.5 \%$ FCS (for $16 \mathrm{HBE}$ cells) or basal medium (BEBM; Lonza)/0.5\% FCS (for PBECs). Methods for epithelial resistance measurements, western blotting and immunofluorescence are described in the online supplementary material.

\section{Preparation of CSE}

Cigarette smoke extract (CSE) was prepared as described previously [17]. In short, Kentucky 2R4F research-reference cigarettes (The Tobacco Research Institute, University of Kentucky, Lexington, KY, USA) were used without filter. Smoke from two cigarettes was bubbled through $25 \mathrm{~mL}$ medium (100\% CSE). The extract was prepared freshly, sterilised using a $0.22 \mu \mathrm{m}$ filter and used within $30 \mathrm{~min}$.

\section{Stimulation of epithelial cells}

Cells were pretreated with or without specific EGFR tyrosine kinase inhibitor AG1478 (2 $\mu$ M; Calbiochem; La Jolla, CA, USA), calpain inhibitor II (ALLM, $10 \mu \mathrm{M}$; Sigma-Aldrich, St Louis, MO, USA) or vehicle (dimethylsulfoxide (DMSO)) for $30 \mathrm{~min}$. Subsequently, cells were treated with 5\% CSE, epidermal growth factor (EGF) $\left(10 \mathrm{ng} \cdot \mathrm{mL}^{-1}\right.$; Sigma-Aldrich) or vehicle (medium/DMSO) for 0-24 $\mathrm{h}$ and monitored by ECIS, fixed for immunofluorescent staining or harvested for cell lysate preparation. Cell viability was evaluated by Trypan blue staining. $5 \%$ CSE did not affect cell viability (data not shown).

\section{ECIS}

Electrical properties of confluent or wounded epithelium were measured using ECIS (Applied Biophysics, Troy, NY, USA) as described previously $[18,19]$. Upon inoculation, resistance and capacitance were measured at $400 \mathrm{~Hz}$ and $40 \mathrm{kHz}$, respectively. Cells were wounded immediately after addition of CSE/vehicle by electroporation ( $5 \mathrm{~V}, 40 \mathrm{kHz}, 30 \mathrm{~s})$.

\section{Statistical analysis}

Data were analysed using the nonparametric rank sum MannWhitney U-test for analysis between subject groups and the t-test for paired observations to compare conditions within groups or the $16 \mathrm{HBE}$ cell line. Two-way ANOVA was used to test for significant differences between the control and treatment time curves in the epithelial resistance measurements. 


\section{RESULTS}

CSE induces a transient decrease in electrical resistance of 16HBE cells

We first examined the effect of CSE on barrier function in a monolayer of $16 \mathrm{HBE}$ cells. Addition of CSE induced a substantial transient fall in low-frequency resistance, the most sensitive parameter to monitor barrier tightness $[18,19]$. Resistance levels started to decline immediately, with a maximal effect between 4
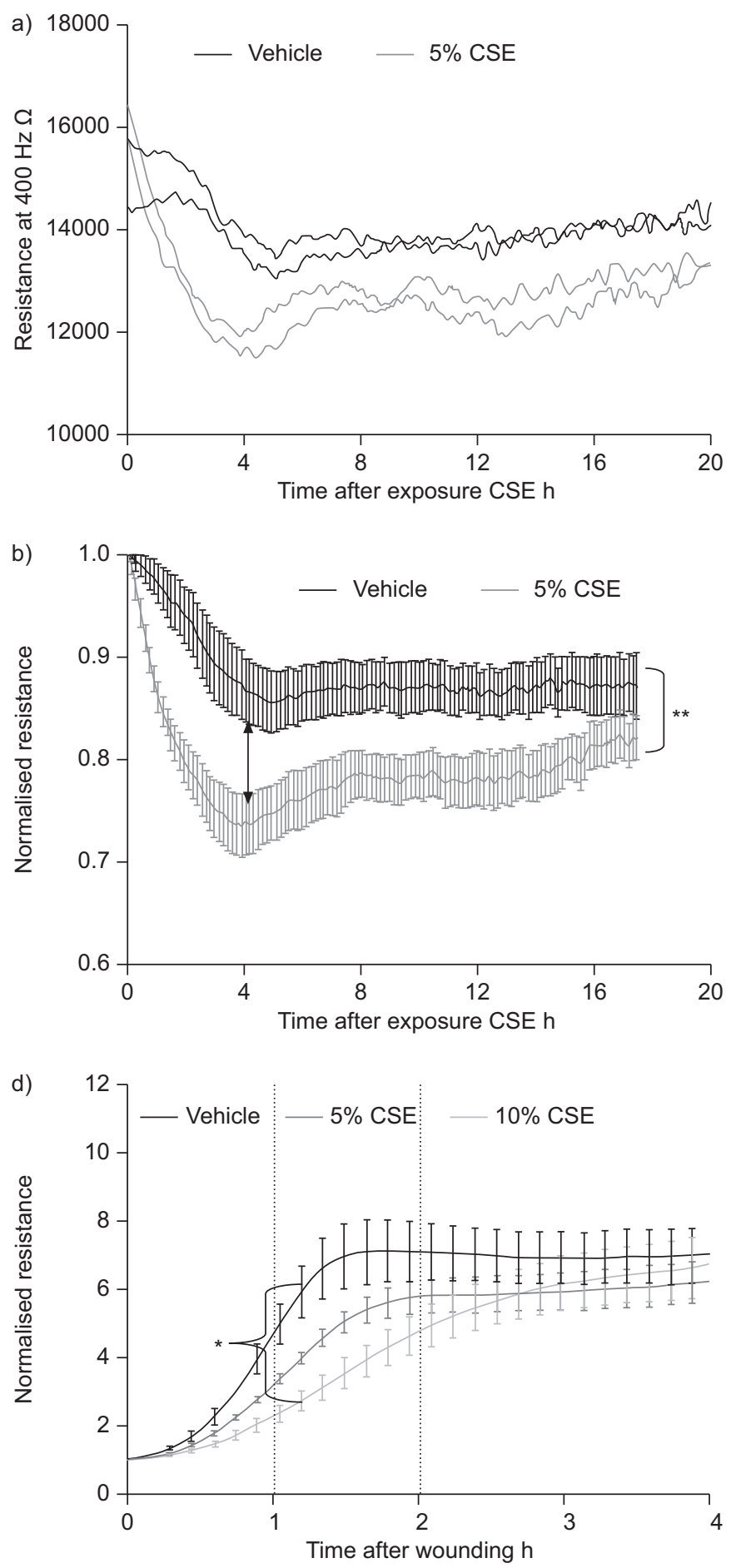

and $6 \mathrm{~h}$ (maximal mean \pm SEM decrease $26 \pm 3 \% ; n=5$ ), followed by a slow return to control values over $\sim 15 \mathrm{~h}$ (fig. $1 \mathrm{a}$ and $\mathrm{b}$ ). Resistance of the vehicle-treated wells also decreased over 4-6 h (maximal mean \pm SEM inhibition $14 \pm 3 \%$ ), but a significantly stronger decrease was observed with CSE $(n=5 ; p=0.011)$. We additionally measured the high-frequency capacitance, which is sensitive to changes in cell-matrix contacts, but relatively insensitive to changes in cell-cell contacts [18, 19]. Here, CSE

FIGURE 1. Effects of cigarette smoke extract (CSE) on electrical resistance in $16 \mathrm{HBE}$ cells. $16 \mathrm{HBE}$ cells were seeded in duplicates in electric cell substrate impedance sensing (ECIS) arrays, grown for 3-4 days, placed at low serum, exposed to $5 \%$ or $10 \%$ CSE or vehicle and monitored for $48 \mathrm{~h}$ or immediately wounded by electroporation. Resistance was measured at $400 \mathrm{~Hz}$ and capacitance was measured at $40 \mathrm{kHz}$ using ECIS. a) Absolute resistance values of a representative experiment are shown. b) Normalised resistance values (mean \pm SEM) are shown $(n=5)$. Values were normalised to the levels prior to the addition of CSE/vehicle. ${ }^{* *}: p<0.01$ between the control and CSE curve, two-way ANOVA. c) Normalised capacitance values (mean \pm SEM) are shown $(n=5)$. d) Normalised resistance values (mean \pm SEM) are shown $(n=3)$. Values were normalised to the levels immediately upon wounding. ${ }^{*}: p<0.05$ between the control and 10\% CSE curve, two-way ANOVA. e) Normalised capacitance values (mean \pm SEM) are shown $(n=3)$.
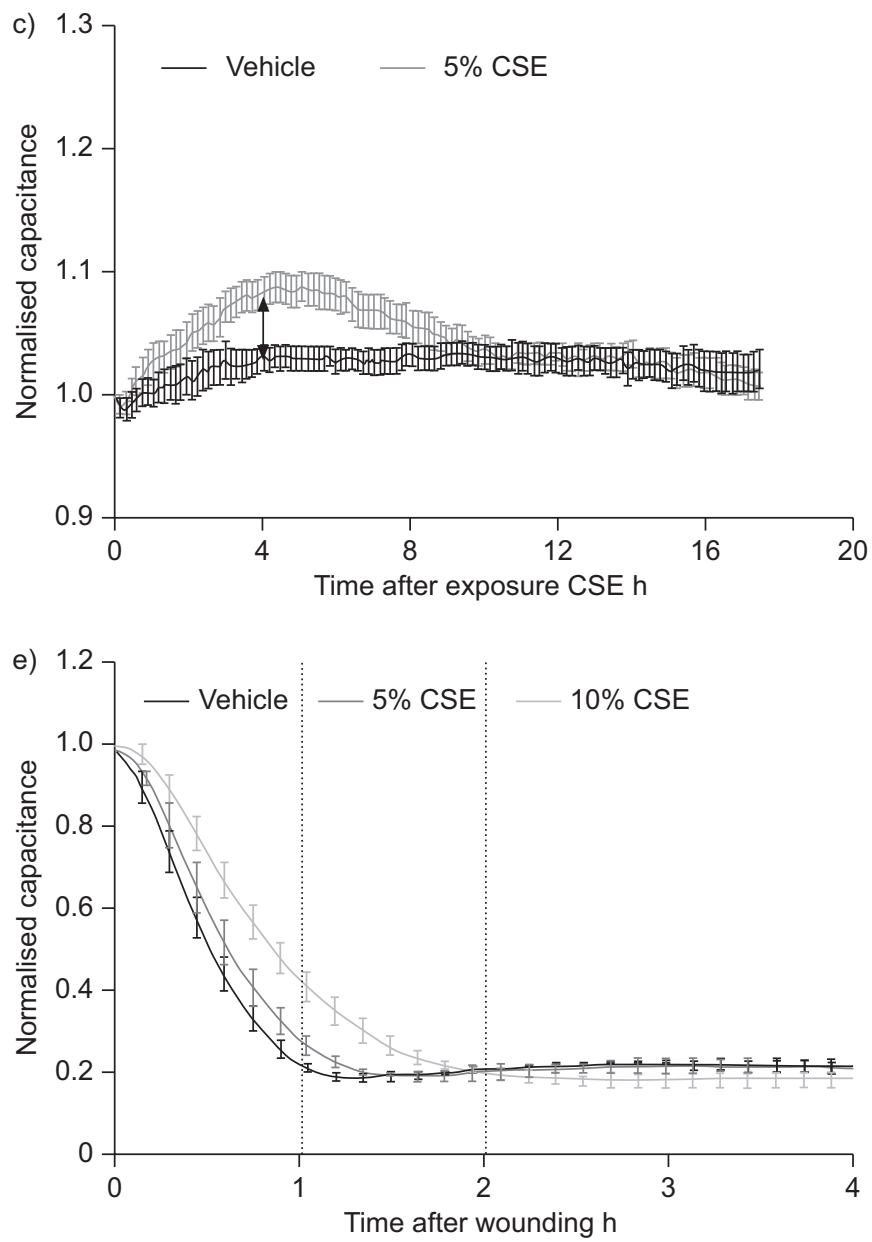
exerted less pronounced effects (mean \pm SEM maximal increase $9 \pm 1 \%$ ), indicating that cell-matrix contacts were affected to a smaller extent than cell-cell contacts (fig. 1c).

\section{CSE delays epithelial regeneration and reconstitution of cell-cell contacts of 16 HBE cells upon wounding by electroporation}

In addition to its effects on 16HBE barrier function, CSE impaired epithelial barrier reconstitution after wounding by electroporation. Using this type of wounding, the epithelial monolayer is able to recover within a few hours, as a result of cell migration/ spreading, and not from proliferation [19]. Barrier function of the 16HBE cells completely recovered within $2 \mathrm{~h}$ (fig. 1d). Cells repopulated the electrode in $1 \mathrm{~h}$, as reflected by the stabilisation of high-frequency capacitance (fig. 1e). CSE markedly delayed the recovery of epithelial resistance upon wounding. While a trend towards reduced epithelial resistance was observed when CSE was added in a concentration of 5\%, the addition of $10 \%$ CSE significantly impaired recovery of the epithelial barrier (fig. 1d). Again, CSE (5 and 10\%) displayed less dramatic effects on highfrequency capacitance than on low-frequency resistance. 1-2 hrs after wounding, low-frequency resistance was still markedly reduced compared with the control-treated wells, while capacitance values had almost completely returned to levels of the confluent monolayer for both 5 and 10\% CSE (fig. 1e). This indicates that CSE affects the reconstitution of epithelial cell-cell contacts rather than affecting epithelial migration, spreading and/or attachment upon wounding.

\section{CSE-induced defects in barrier function are reversed by inhibition of EGFR activity}

Since previous studies proposed a role for EGFR-dependent signalling in CSE-induced redistribution of TJ proteins [11], and that increased EGFR activity has been implicated in COPD [20], we next investigated whether EGFR activity was involved in the CSE-induced defects in epithelial barrier function. Treatment of the 16HBE cells with the EGFR tyrosine kinase inhibitor AG1478 significantly and almost completely prevented the CSE-induced changes in low-frequency resistance (fig. 2a and c). Similarly, AG1478 attenuated the CSE-induced increase in high-frequency capacitance, which was significant within 30-120 min (fig. $2 \mathrm{~b}$ and $\mathrm{d}$, but not at the time point where CSE exerted its strongest effects, i.e. $4 \mathrm{~h}$ after addition of CSE (fig. 1c).

In line with the involvement of EGFR activity in CSE-induced barrier dysfunction, we found that CSE increased phospho-EGFR
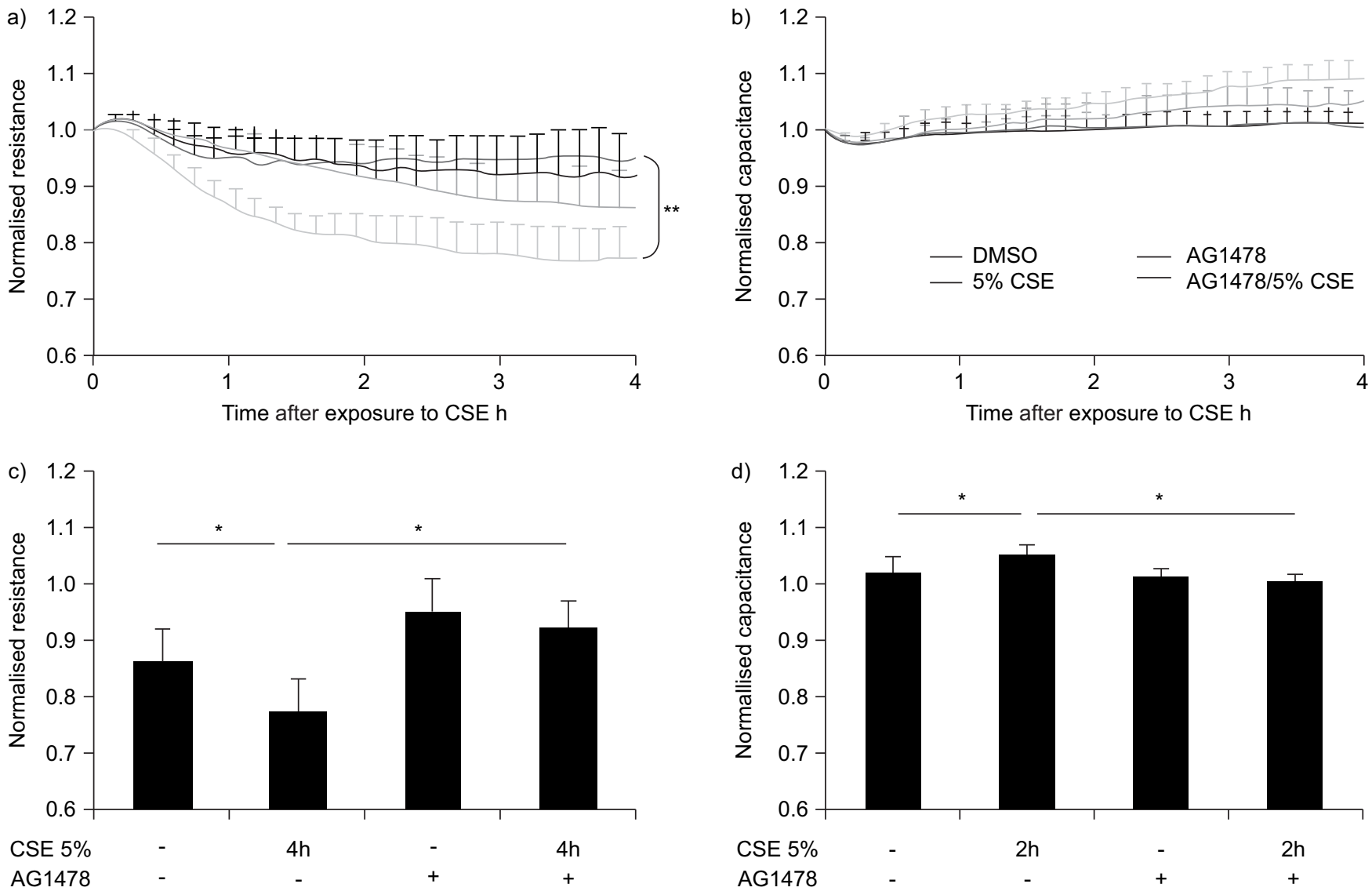

FIGURE 2. The effects of cigarette smoke extract (CSE) on barrier function in $16 \mathrm{HBE}$ cells are dependent on epidermal growth factor receptor activity. $16 \mathrm{HBE}$ cells were seeded in duplicates in electric cell substrate impedance sensing arrays, grown for 3-4 days, placed at low serum, pretreated for 30 min with or without AG1478 (1 $\mu \mathrm{M}$ ) and exposed to $5 \%$ CSE or vehicle. Resistance was measured at $400 \mathrm{~Hz}$ and capacitance was measured at $40 \mathrm{kHz}$. Values were normalised to the levels prior to the addition of CSE/vehicle. $a$ and $c)$ Normalised resistance values (mean \pm SEM) are shown $(n=5) . * *: p<0.01$ between the indicated curves (CSE and CSE+AG1478), two-way ANOVA; *: $p<0.05$ between the indicated values. $b$ and $d)$ Normalised capacitance values (mean \pm SEM) are shown $(n=5)$. *: $p<0.05$ between the indicated values. 
(Tyr 1173) as well as EGFR downstream signalling molecule phospho-ERK-1/2, but not total EGFR levels, at $6 \mathrm{~h}$ (fig. 3a and b). Furthermore, addition of EGF transiently decreased epithelial resistance, with a maximum effect around 4-6 h, after which the cells recovered slowly (fig. $3 \mathrm{c}$ and d).

\section{CSE induces EGFR-dependent disassembly of TJs}

To underscore the mechanism of CSE-induced barrier dysfunction, we studied whether the changes in barrier function were paralleled by changes in intercellular junctions. We observed that extra- and intracellular TJ components, i.e. ZO-1 and occludin, respectively, were localised to intercellular junctions under resting conditions, forming a continuous ring (fig. 4a). Upon CSE exposure, staining for ZO-1 (fig. 4a) and also, to a lesser extent, occludin (fig. $4 \mathrm{~b}$ ) became more fragmented. The presence of AG1478 partially reversed these effects, resulting in more circumferential staining of ZO-1 and occludin (fig. 4a and $b$ ). In agreement with the hypothesis that EGFR activity plays a role in CSE-induced TJ disassembly, EGF induced delocalisation of ZO-1 and occludin (fig. $4 \mathrm{a}$ and b).

Next, we investigated whether CSE and EGF also affected total levels of occludin and ZO-1. CSE induced partial ZO-1 cleavage, as demonstrated by the appearance of a smaller fragment $(\sim 192 \mathrm{kDa})$, and partial cleavage of occludin from its full length form $(\sim 65 \mathrm{kDa})$ into smaller cleavage fragments $(\sim 45$ and $\sim 30 \mathrm{kDa}$; fig. 4c-e). We did not observe cleavage of adherens junction (AJ) protein E-cadherin (data not shown). The presence of AG1478 reduced the appearance of smaller ZO-1 and occludin fragments (fig. 4c-e). Previously, activation of the endogenous cysteine protease calpain has been implicated in EGFR signalling [21] and calpain has been described to cleave junctional proteins [22]. The calpain inhibitor II also reduced the CSE-induced cleavage of occludin and ZO-1 into smaller fragments. Thus, exposure to CSE appears to disrupt TJs in an EGFR-dependent manner, and our data suggest this to involve calpain-mediated cleavage of TJ proteins. a)

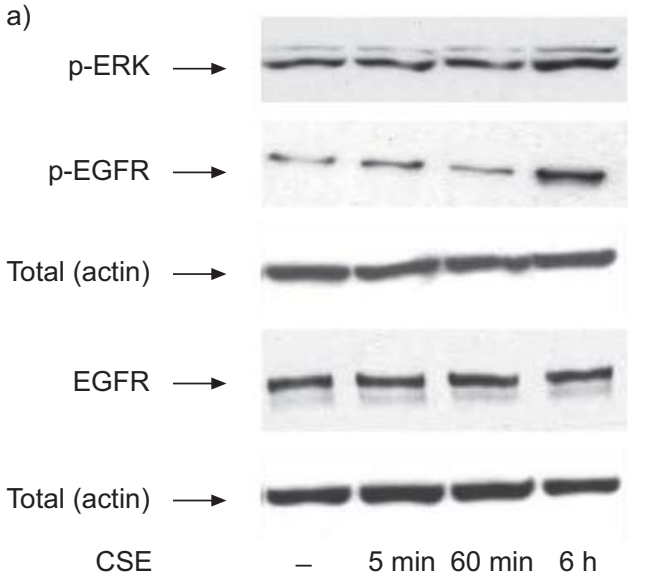

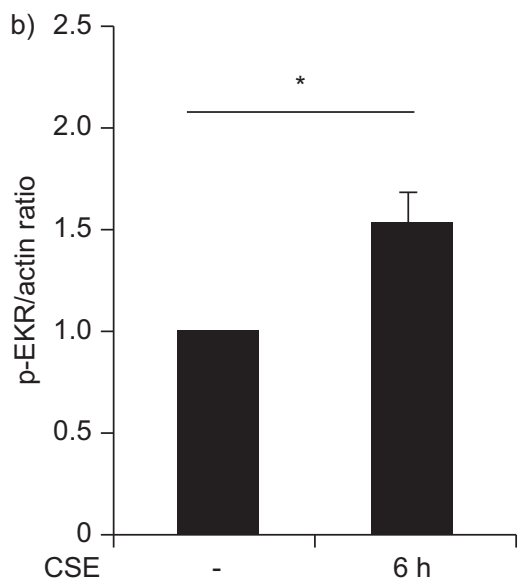

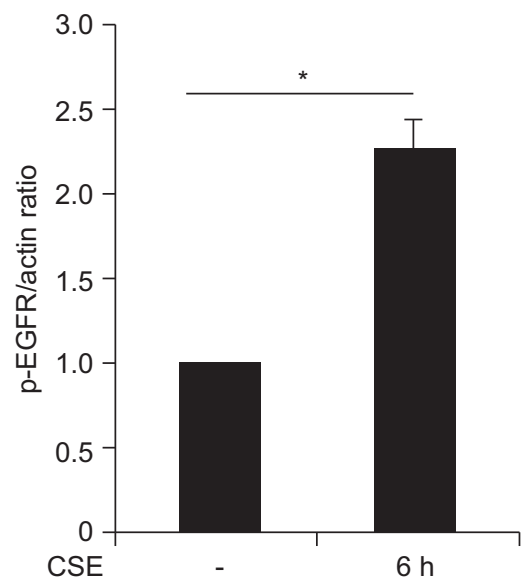

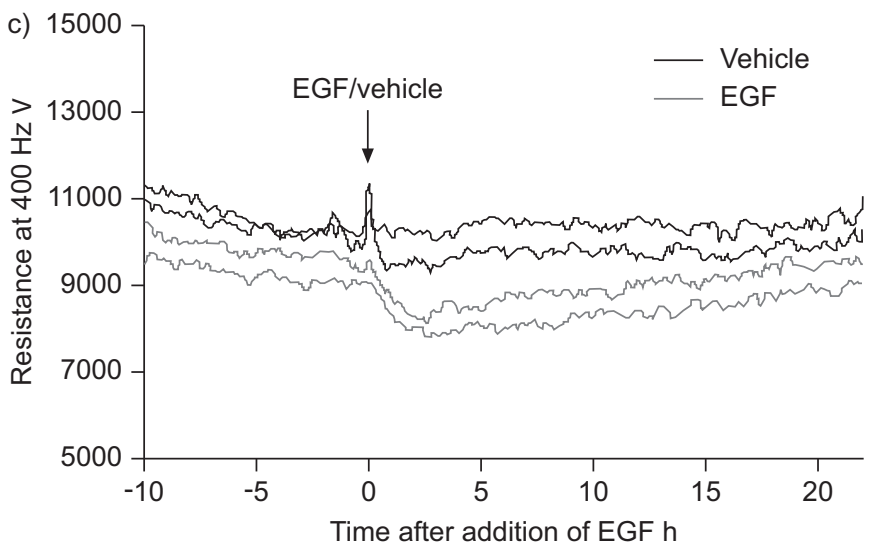

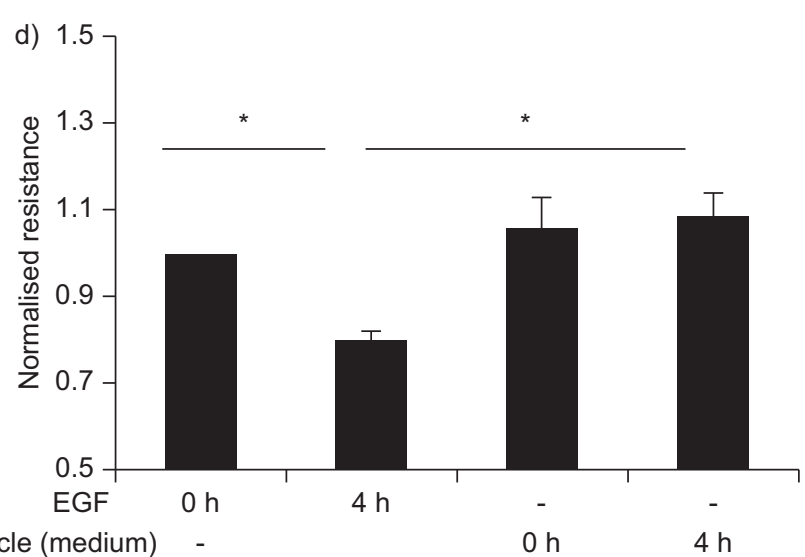

FIGURE 3. Cigarette smoke extract (CSE) induces epidermal growth factor receptor (EGFR)-dependent signalling and epidermal growth factor (EGF) affects electrical resistance of $16 \mathrm{HBE}$ cells. $16 \mathrm{HBE}$ cells were seeded in duplicates in 24-well plates or electric cell substrate impedance sensing arrays, grown to confluence for 3-4 days, placed at low serum and stimulated with or without $5 \%$ CSE or EGF $\left(10 \mathrm{ng} \cdot \mathrm{mL}^{-1}\right)$ for $5 \mathrm{~min}$ to $24 \mathrm{~h}$, as indicated. a) Total cell lysates were prepared and phospho-extracellular signal-related kinase (phospho-ERK), phospho-EGFR and EGFR were detected by western blotting (arrows). Actin was used as loading control. Representatives of 4 independent experiments are shown. b) Densitometry was performed and phospho-ERK and phospho-EGFR levels were related to actin. Ratios (mean \pm SEM; $\mathrm{n}=4$ ) are depicted. c) Absolute resistance values of a representative experiment are shown. d) Resistance values normalised to the levels prior to the addition of EGF/vehicle are shown (mean \pm SEM; $\mathrm{n}=3$ ). *: $\mathrm{p}<0.05$ between the indicated values. 
a)

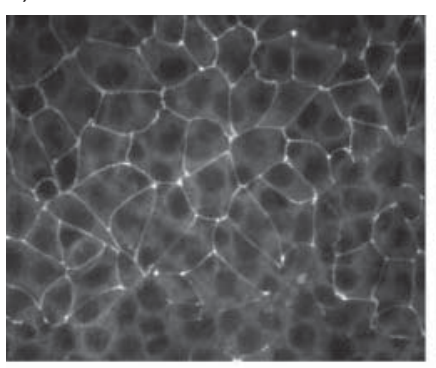

Control

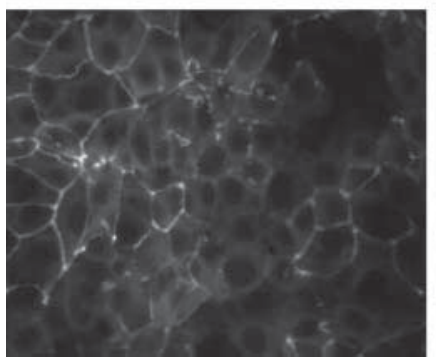

CSE 5\%
ZO-1

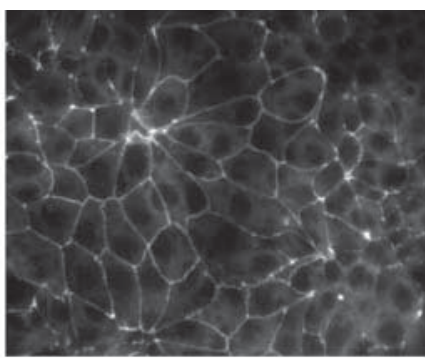

CSE+AG1478

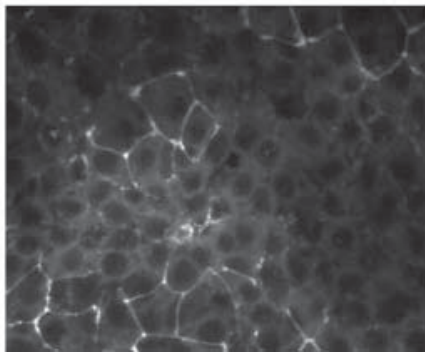

EGF b)

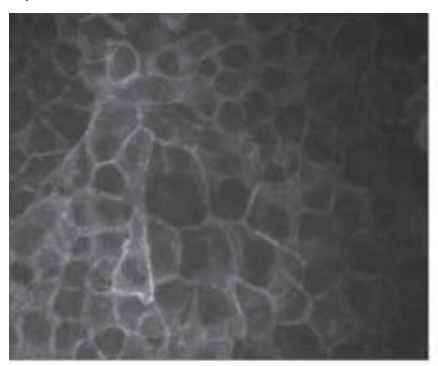

Control

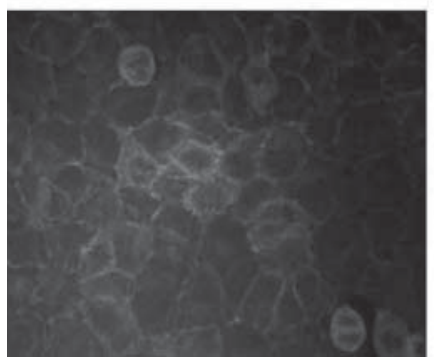

CSE $5 \%$
Occludin

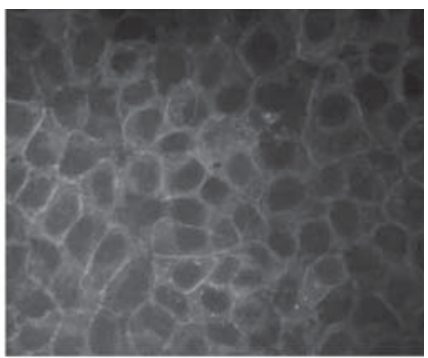

CSE+AG1478

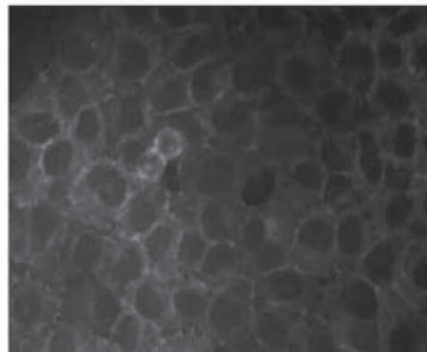

EGF c)

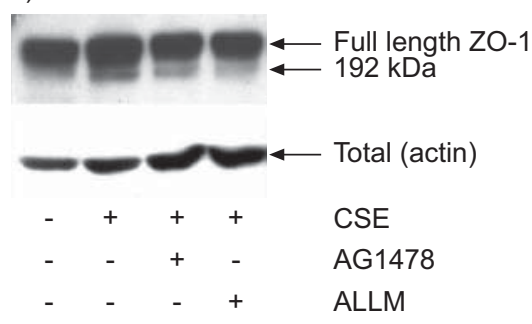

d)

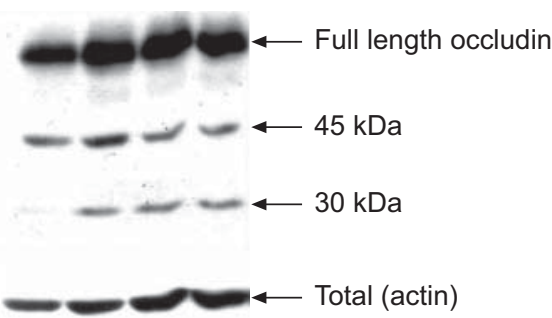

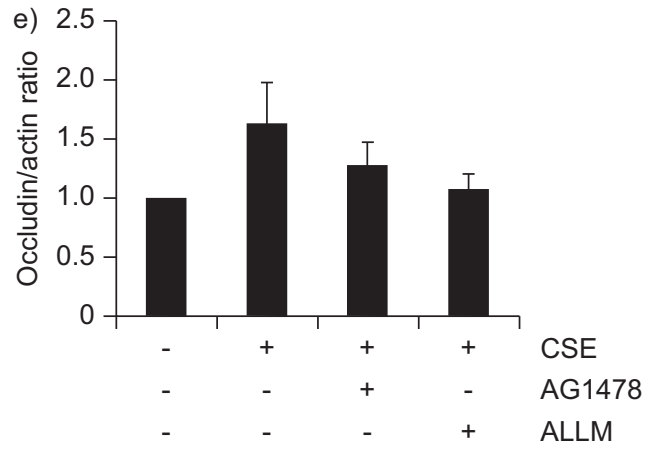

FIGURE 4. Cigarette smoke extract (CSE) and epidermal growth factor (EGF) induce disassembly of tight junctions. 16HBE cells were cultured in chamber slides or 24well plates for 3 days, placed at low serum, pretreated for $1 \mathrm{~h}$ with and without AG1478 $(1 \mu \mathrm{M})$ or ALLM $(10 \mu \mathrm{M})$ and incubated with or without CSE (5\%) or EGF $\left(10 \mathrm{ng} \cdot \mathrm{mL}^{-1}\right)$ for $4 \mathrm{~h}$. a) Zona occludens-1 (ZO-1) was detected by immunofluorescence staining. b) Occludin was detected by immunofluorescence staining. c) Total cell lysates were prepared and ZO-1 was detected by western blotting (arrows). Representatives of four independent experiments are shown. d) Total cell lysates were prepared and occludin was detected by western blotting (arrows). Actin was used as loading control. e) Densitometry was performed on the $30 \mathrm{kDa}$ fragment of occludin and levels were related to actin; ratios (mean \pm SEM; $n=4$ ) are depicted.

\section{CSE induces EGFR-dependent defects in barrier function of PBEC from nonsmokers and healthy smokers}

Next, we were interested in the effects of CSE in primary epithelium and possible differences between nonsmoking individuals and current smokers, in order to assess whether epithelial abnormalities are present upon long-term smoking. PBECs showed considerably lower resistance levels upon establishment of the monolayer than $16 \mathrm{HBE}$ cells, with no significant differences between nonsmokers and smokers (mean \pm SEM levels $4,257 \pm 1,422 \Omega$ and $5,238 \pm 1,350 \Omega$, respectively). Exposure to CSE induced a $\sim 50 \%$ decrease in epithelial barrier function in both nonsmokers and smokers (fig. $5 \mathrm{a}$ and $5 b$ ), to levels of $1,937 \pm 563 \Omega$ and $2,942 \pm 955 \Omega$, respectively. The onset of the CSE-induced barrier dysfunction was delayed when compared to $16 \mathrm{HBE}$ cells, with the largest effect between 12-18 h, without recovery until 25-35 h after CSE exposure. No significant differences were observed between the two groups.
Next, we tested for the involvement of EGFR activity. Similar to $16 \mathrm{HBE}$ cells, AG1478 significantly reduced the CSE-induced defect in PBEC barrier function at 9-28 h (data shown for $12 \mathrm{~h}$, the time point where most pronounced effects of CSE were observed; fig. 5c), and CSE was no longer able to significantly reduce epithelial resistance in the presence of AG1478. AG1478 also increased epithelial resistance in the absence of CSE; however, this effect did not reach statistical significance until $30 \mathrm{~h}$ upon its addition. Furthermore, AG1478 abolished the effect of CSE on high-frequency capacitance, although this effect of CSE was less pronounced than on low-frequency resistance, and only significant at 3-5 h (fig. 1 of the online supplementary material).

Additionally, we studied the response of PBECs to wounding by electroporation and observed that these cells regenerated more slowly than $16 \mathrm{HBE}$ cells, as it took cells $\sim 3 \mathrm{~h}$ to restore 
PBEC healthy donors
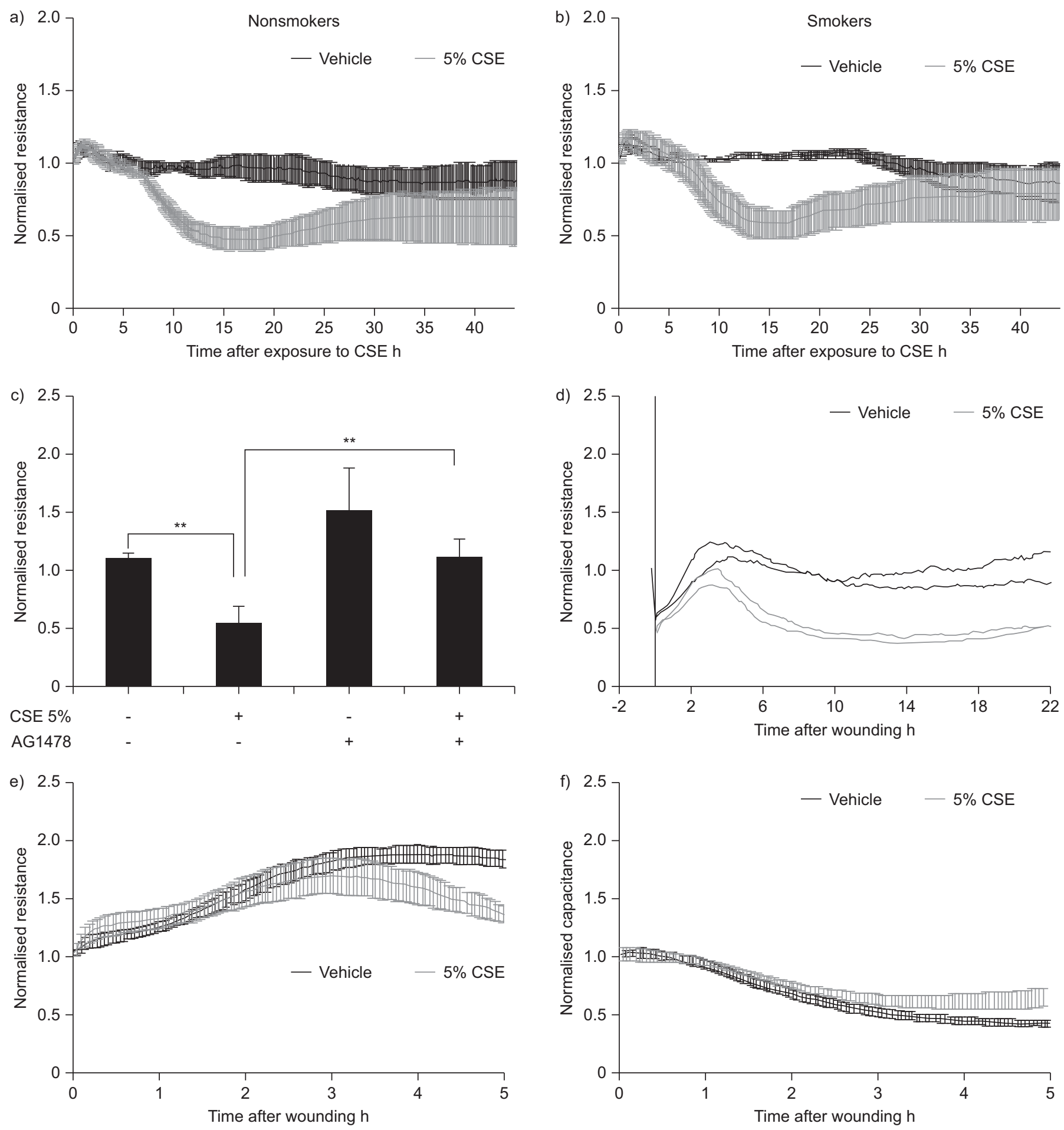

FIGURE 5. Primary bronchial epithelial cells (PBECs) from nonsmokers and smokers were seeded in duplicates in electric cell substrate impedance sensing arrays, grown to confluence for 3-4 days, growth factor/hormone-deprived overnight, exposed to $5 \%$ cigarette smoke extract (CSE) or vehicle and monitored for $48 \mathrm{~h}$ or immediately wounded by electroporation. Resistance was measured at $400 \mathrm{~Hz}$ and capacitance was measured at $40 \mathrm{kHz}$. Values were normalised to the levels prior to the addition of CSE/vehicle. When enough cells were available, cells were pretreated with or without AG1478 before CSE exposure. a) Normalised resistance values (mean \pm SEM) of PBECS from nonsmokers $(n=3)$. Values were normalised to the levels prior to the addition of CSE/vehicle. b) Normalised resistance values (mean \pm SEM) of PBECs from smokers $(n=4)$. c) Normalised resistance values (mean \pm SEM) (PBECs from both smokers and nonsmokers; $n=4)$. ${ }^{*}: p<0.01$ between the indicated values. $d$ ) Normalised resistance values of PBECs from a smoker (representative). Values were normalised to the levels prior to wounding. e) Normalised resistance values (mean \pm SEM) of PBECs from smokers $(n=3)$. Values were normalised to the levels immediately upon wounding. f) Normalised capacitance values (mean \pm SEM) of PBECs from smokers $(n=3)$. 
their barrier function (shown for healthy smoker epithelium; fig. $5 \mathrm{~d}$ and e). Again, we did not observe differences between epithelial cells from smokers and nonsmokers (fig. 2 of the online supplementary material). Exposure to CSE reduced the resistance of the newly established monolayer, with a further decline once the resistance values in untreated cells were stabilised (fig. 5e). Effects on high-frequency capacitance were again less pronounced (fig. 5f).

\section{CSE induces similar defects in barrier function of PBECs from COPD patients}

To further evaluate the relevance of our findings, we used PBECs from three patients with severe COPD (all ex-smokers). Monolayers of these cells established a similar low-frequency resistance to that of healthy PBECs, i.e. 3,281 $\pm 926 \Omega$. In addition, CSE caused an equal $\sim 50 \%$ decrease in barrier function (fig. 6a), resulting in levels of $1,516 \pm 163 \Omega$. In further agreement with the healthy PBECs, low-frequency resistance was affected more markedly by CSE than high-frequency capacitance (data not shown).

Furthermore, the response to wounding by electroporation was similar in PBECs from COPD patients and healthy PBECs, with resistance levels returning to their original values within $\sim 3 \mathrm{~h}$. Again, CSE reduced the low-frequency resistance of the
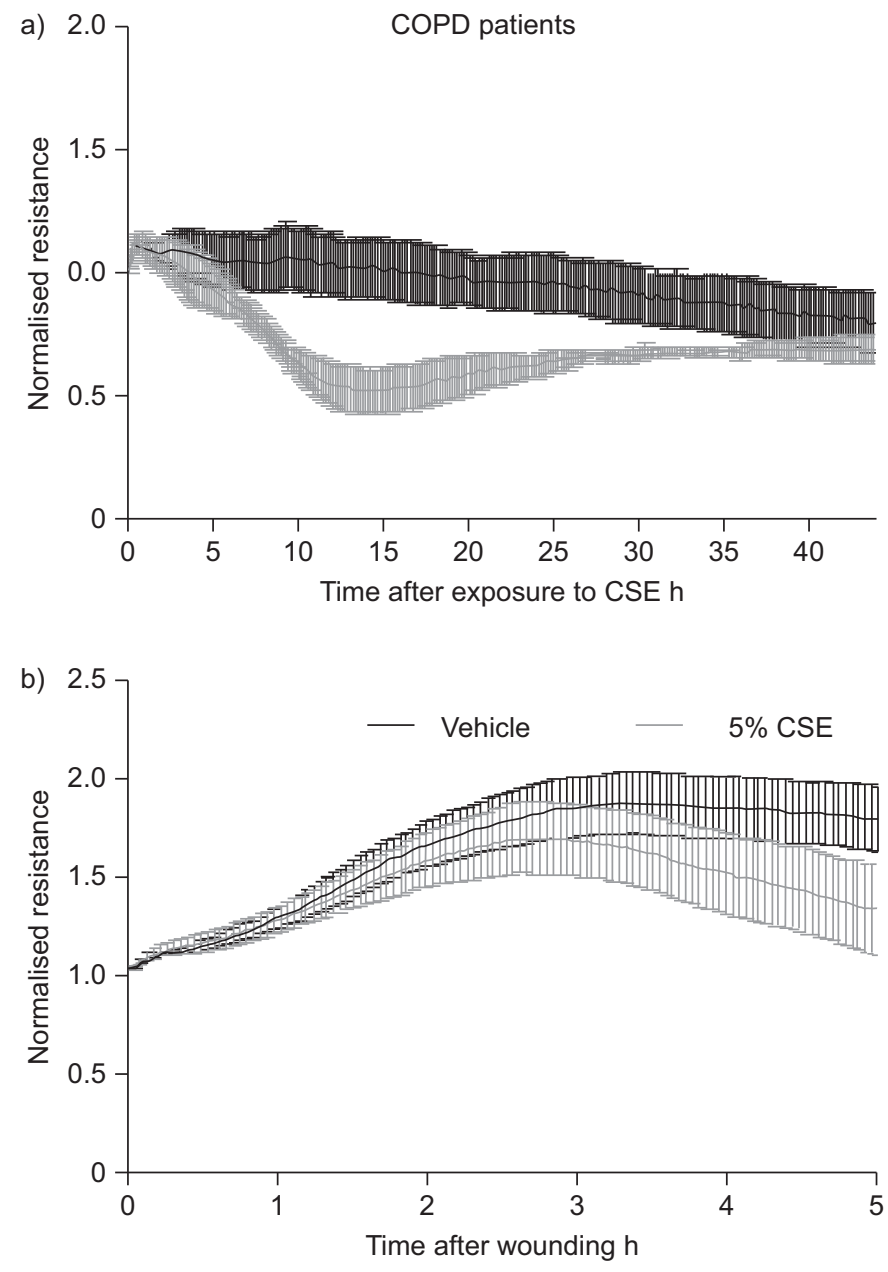

newly formed monolayer upon wounding (fig. 6b), with less pronounced effects on high-frequency capacitance (fig. 6c).

Together, our data indicate that CSE exposure impairs the integrity of airway epithelium, mainly by disruption of intercellular contacts, with a similar pattern in COPD and healthy epithelium.

\section{DISCUSSION}

Epithelial remodelling and aberrant epithelial repair responses may disturb mucosal barrier function in COPD. Cigarette smoke is known to increase epithelial permeability, although the underlying mechanism has remained incompletely understood. Our study shows that CSE causes a transient, but substantial, decrease in airway epithelial barrier function in both 16HBE cells and PBECs. This effect appears dependent on EGFR activation, and we provide evidence for a novel mechanism of barrier dysfunction involving calpain-mediated disruption of TJs. Furthermore, CSE decreased restoration of $16 \mathrm{HBE}$ cell-cell contacts during wound regeneration and attenuated barrier function of PBECs upon wounding, with no striking differences between COPD patients and healthy controls. Our data may have important implications, e.g. for exacerbations in smokers with COPD, since reduced epithelial

FIGURE 6. Bronchial epithelial cells were derived from bronchial brushings in Global Initiative for Chronic Obstructive Lung Disease stage IV chronic obstructive pulmonary disease patients. Cells were seeded in duplicates in electric cell substrate impedance sensing arrays, grown to confluence for 3-4 days, growth factor/hormone-deprived overnight, exposed to $5 \%$ CSE or vehicle and monitored for $48 \mathrm{~h}$ or immediately wounded by electroporation. Resistance was measured at $400 \mathrm{~Hz}$ and capacitance was measured at $40 \mathrm{kHz}$. a) Normalised resistance values (mean \pm SEM) are shown $(n=3)$. Values were normalised to the levels prior to the addition of CSE or vehicle. b) Normalised resistance values (mean \pm SEM) are shown $(n=3)$. Values were normalised to the levels immediately upon wounding. c) Normalised capacitance values (mean \pm SEM) are shown $(n=3)$. Values were normalised to the levels immediately upon wounding.

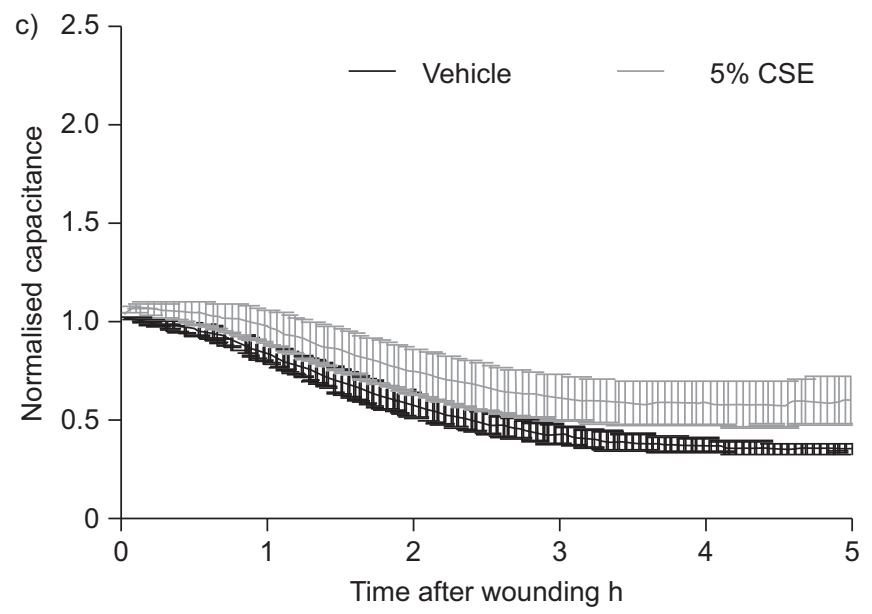


integrity may facilitate the transport of viruses and bacteria that make an important contribution to COPD exacerbations.

CSE affected epithelial resistance similarly in PBECs from COPD patients, healthy smokers and nonsmokers. Considering the role of EGFR in the CSE-induced defects, this is in line with our observation that EGFR levels did not differ between the subject groups (fig. 3 of the online supplementary material). The effects in PBECs were stronger and more sustained than in 16HBE cells. It is possible that PBECs are more susceptible to CSE-induced damage as result of weaker intercellular contacts [19]. Primary cells are able to form a tight barrier when cultured at an airliquid interface (ALI), allowing for differentiation into polarised mucociliary epithelium [23]. Unfortunately, the ECIS system does not allow for measurements under ALI conditions. We have previously shown that culturing under ALI conditions substantially increases membrane expression of junctional proteins in PBECs, resulting in an epithelial resistance that equals the levels in 16HBE cells [24]. Therefore, we consider $16 \mathrm{HBE}$ cells as a reliable model when studying epithelial barrier function [19]. Indeed, 16HBE cells have been reported to polarise and form functional AJs and TJs under submerged conditions [25]. In both $16 \mathrm{HBE}$ cells and PBECs, EGFR activity appeared to contribute to the CSE-induced defects. Previously, EGFR activity has been shown to induce delocalisation of junctional proteins and calpain activation [21, 26, 27], which may lead to degradation of TJ proteins [28]. Our data support a role for EGFR in the CSE-induced disassembly of TJs, since the EGFR tyrosine kinase inhibitor AG1478 abrogated the CSE-induced defect on barrier function. Furthermore, CSE increased EGFR phosphorylation in $16 \mathrm{HBE}$ cells. Accordingly, activation of EGFR by its ligand EGF transiently decreased epithelial barrier function, and both CSE and EGF induced delocalisation as well as cleavage of the TJ proteins occludin and ZO-1 in 16HBE cells.

Several mechanisms may underlie this CSE-induced EGFR activation. First, increased access of apically secreted EGF ligands to basolaterally localised EGFR could be involved upon opening of tight junctions [29]. However, this does not seem to be the case, as EGFR activity itself was required for the disruption of TJs. Alternatively, smoke has been described to activate A disintegrin and metalloproteinase (ADAM)17, the main sheddase of the EGFR ligand transforming growth factor (TGF)- $\alpha$ [30]. We observed that CSE induces ADAM17 and EGFRdependent interleukin- 8 production in 16HBE cells (fig. 4 of the online supplementary material), suggesting that CSE can indeed activate ADAM17. However, we were not able to detect TGF- $\alpha$ upon CSE exposure. Moreover, the presence of ADAM17/10 inhibitor GW280264 did not prevent the CSE-induced defect on barrier function nor the CSE-induced activation of EGFR (data not shown), rendering a role for ADAM17 less likely. Thus, a more plausible explanation might be the induction of Src kinase activity and subsequent ligand-independent EGFR phosphorylation, which has been described in A549 cells [31] and we will explore this possibility in future studies.

In addition to its effects on cell-cell contacts, EGFR activity can affect cell-matrix contacts by induction of calpain activity and cleavage of fibronectin and/or focal adhesion component talin [32-34]. In our setting, the modest CSE-induced increase in high-frequency capacitance reflected effects on cell-matrix contacts, and the presence of AG1478 abrogated these effects. In both
16HBE cells and PBECs, we observed more pronounced effects on low-frequency resistance than on high-frequency capacitance, suggesting that cigarette smoke-induced EGFR activity affects intercellular contacts more than cell-matrix contacts.

Our data show that CSE also affects the reconstitution of intercellular contacts in response to wounding, causing a delay in the recovery of barrier function in $16 \mathrm{HBE}$ cells. In PBECs, CSE impaired barrier function upon re-establishment of the monolayer rather than during its recovery. We do not have an explanation for the later onset of CSE-induced defects in PBECs. It may well be that this involves differential effects of CSE on intracellular signalling and/or on cell architecture in the two cell types. Again, we did not observe significant differences in the epithelial responses of nonsmokers and healthy smokers, in either presence or absence of CSE. This indicates that long-term smoking does not induce changes that remain present after the culture procedure, e.g. with respect to proteins involved in epithelial regeneration. Furthermore, PBECs from COPD patients did not show striking differences with regard to their responses to wounding and/or CSE exposure when compared to healthy PBECs. However, we cannot exclude the possibility that there are differences in ALIpolarised PBECs, between those in COPD patients and those in healthy individuals. It will be of interest to further explore possible intrinsic differences in COPD epithelium in a separate, future study including larger numbers of COPD patients and epithelial cells derived from bronchial brushings in healthy donors matched for age and smoking history.

In conclusion, we demonstrate that cigarette smoke induces EGFR-dependent disruption of TJs and reduces airway epithelial integrity upon wounding, possibly through a mechanism involving the endogenous protease calpain. We propose that cigarette smoking may have important implications for bacterial and viral infections in COPD, both by facilitating the access of pathogens as well as impairing recovery of the mucosal immune barrier upon pathogen-induced damage. Thus, targeting the EGFR may provide new avenues for the treatment of COPD exacerbations.

\section{STATEMENT OF INTEREST}

A statement of interest for this study can be found at www.erj. ersjournals.com/site/misc/statements.xhtml

\section{ACKNOWLEDGEMENTS}

We thank H.G. de Bruin (University Medical Center Groningen, Groningen, the Netherlands) for his help with the immunodetection experiments.

\section{REFERENCES}

1 Kato Y, Hirano T, Yoshida K, et al. Frequent loss of E-cadherin and/or catenins in intrabronchial lesions during carcinogenesis of the bronchial epithelium. Lung Cancer 2005; 48: 323-330.

2 Broekema M, Ten Hacken NH, Volbeda F, et al. Airway epithelial changes in smokers but not in ex-smokers with asthma. Am J Respir Crit Care Med 2009; 180: 1170-1178.

3 Takeyama K, Jung B, Shim JJ, et al. Activation of epidermal growth factor receptors is responsible for mucin synthesis induced by cigarette smoke. Am J Physiol Lung Cell Mol Physiol 2001; 280: L165-L172. 
4 Hogg JC. Bronchial mucosal permeability and its relationship to airways hyperreactivity. Eur J Respir Dis Suppl 1982; 122: 17-22.

5 Gangl K, Reininger R, Bernhard D, et al. Cigarette smoke facilitates allergen penetration across respiratory epithelium. Allergy 2009; 64: 398-405.

6 Rusznak C, Sapsford RJ, Devalia JL, et al. Cigarette smoke potentiates house dust mite allergen-induced increase in the permeability of human bronchial epithelial cells in vitro. Am J Respir Cell Mol Biol 1999; 20: 1238-1250.

7 Sethi S, Murphy TF. Infection in the pathogenesis and course of chronic obstructive pulmonary disease. N Engl J Med 2008; 359: 2355-2365

8 Shaykhiev R, Otaki F, Bonsu P, et al. Cigarette smoking reprograms apical junctional complex molecular architecture in the human airway epithelium in vivo. Cell Mol Life Sci 2011; 68: 877-892.

9 Olivera DS, Boggs SE, Beenhouwer C, et al. Cellular mechanisms of mainstream cigarette smoke-induced lung epithelial tight junction permeability changes in vitro. Inhal Toxicol 2007; 19: 13-22.

10 Olivera D, Knall C, Boggs S, et al. Cytoskeletal modulation and tyrosine phosphorylation of tight junction proteins are associated with mainstream cigarette smoke-induced permeability of airway epithelium. Exp Toxicol Pathol 2010; 62: 133-143.

11 Petecchia L, Sabatini F, Varesio L, et al. Bronchial airway epithelial cell damage following exposure to cigarette smoke includes disassembly of tight junction components mediated by the extracellular signalregulated kinase 1/2 pathway. Chest 2009; 135: 1502-1512.

12 van der Toorn M, Rezayat D, Kauffman HF, et al. Lipid-soluble components in cigarette smoke induce mitochondrial production of reactive oxygen species in lung epithelial cells. Am J Physiol Lung Cell Mol Physiol 2009; 297: L109-L114.

13 Heijink IH, Kies PM, Kauffman HF, et al. 2007. Down-regulation of E-cadherin in human bronchial epithelial cells leads to epidermal growth factor receptor-dependent Th2 cell-promoting activity. J Immunol, 178: 7678-7685.

14 Kornmann O, Beeh KM, Beier J, et al. Newly diagnosed chronic obstructive pulmonary disease. Clinical features and distribution of the novel stages of the Global Initiative for Obstructive Lung Disease. Respiration 2003; 70: 67-75.

15 Investigative use of bronchoscopy, lavage and bronchial biopsies in asthma and other airways diseases. J Investig Allergol Clin Immunol 1991; 1: 271-277.

16 Lordan JL, Bucchieri F, Richter A, et al. Cooperative effects of Th2 cytokines and allergen on normal and asthmatic bronchial epithelial cells. J Immunol 2002; 169: 407-414.

17 Slebos DJ, Ryter SW, van der Toorn M, et al. Mitochondrial localization and function of heme oxygenase-1 in cigarette smokeinduced cell death. Am J Respir Cell Mol Biol 2007; 36: 409-417.

18 Wegener J, Keese CR, Giaever I. Electric cell-substrate impedance sensing (ECIS) as a noninvasive means to monitor the kinetics of cell spreading to artificial surfaces. Exp Cell Res 2000; 259: 158-166.

19 Heijink IH, Brandenburg SM, Noordhoek JA, et al. Characterisation of cell adhesion in airway epithelial cell types using electric cellsubstrate impedance sensing. Eur Respir J 2010; 35: 894-903.
20 Burgel PR, Nadel JA. Epidermal growth factor receptor-mediated innate immune responses and their roles in airway diseases. Eur Respir J 2008; 32: 1068-1081.

21 Wells A, Gupta K, Chang P, et al. Epidermal growth factor receptor-mediated motility in fibroblasts. Microsc Res Tech 1998; 43: 395-411.

22 Chun J, Prince A. TLR2-induced calpain cleavage of epithelial junctional proteins facilitates leukocyte transmigration. Cell Host Microbe 2009; 5: 47-58.

23 van Wetering S, Zuyderduyn S, Ninaber DK, et al. Epithelial differentiation is a determinant in the production of eotaxin-2 and -3 by bronchial epithelial cells in response to IL-4 and IL-13. Mol Immunol 2007; 44: 803-811.

24 Heijink IH, Postma DS, Noordhoek JA, et al. House dust mitepromoted epithelial-to-mesenchymal transition in human bronchial epithelium. Am J Respir Cell Mol Biol 2010; 42: 69-79.

25 Ehrhardt C, Kneuer C, Fiegel J, et al. Influence of apical fluid volume on the development of functional intercellular junctions in the human epithelial cell line 16HBE14o-: implications for the use of this cell line as an in vitro model for bronchial drug absorption studies. Cell Tissue Res 2002; 308: 391-400.

26 Takai E, Tan X, Tamori Y, et al. Correlation of translocation of tight junction protein Zonula occludens-1 and activation of epidermal growth factor receptor in the regulation of invasion of pancreatic cancer cells. Int J Oncol 2005; 27: 645-651.

27 Rao RK, Basuroy S, Rao VU, et al. Tyrosine phosphorylation and dissociation of occludin-ZO-1 and E-cadherin-beta-catenin complexes from the cytoskeleton by oxidative stress. Biochem J 2002; 368: 471-481.

28 Chun J, Prince A. TLR2-induced calpain cleavage of epithelial junctional proteins facilitates leukocyte transmigration. Cell Host Microbe 2009; 5: 47-58.

29 Carraway CA, Carraway KL. Sequestration and segregation of receptor kinases in epithelial cells: implications for ErbB2 oncogenesis. Sci STKE 2007; 2007: re3.

30 Shao MX, Nakanaga T, Nadel JA. Cigarette smoke induces MUC5AC mucin overproduction via tumour necrosis factoralpha-converting enzyme in human airway epithelial (NCIH292) cells. Am J Physiol Lung Cell Mol Physiol 2004; 287: L420-L427.

31 Khan EM, Lanir R, Danielson AR, et al. Epidermal growth factor receptor exposed to cigarette smoke is aberrantly activated and undergoes perinuclear trafficking. FASEB J 2008; 22: 910-917.

32 Glading A, Chang P, Lauffenburger DA, et al. Epidermal growth factor receptor activation of calpain is required for fibroblast motility and occurs via an ERK/MAP kinase signaling pathway. J Biol Chem 2000; 275: 2390-2398.

33 Franco SJ, Rodgers MA, Perrin BJ, et al. Calpain-mediated proteolysis of talin regulates adhesion dynamics. Nat Cell Biol 2004; 6: 977-983.

34 Frangie C, Zhang W, Perez J, et al. Extracellular calpains increase tubular epithelial cell mobility. Implications for kidney repair after ischemia. J Biol Chem 2006; 281: 26624-26632. 